EDITORIALS

\title{
Vitamin D sufficiency in pregnancy
}

\author{
Better evidence is required to establish optimal levels and need for supplementation
}

\author{
Robyn Lucas associate professor ${ }^{1}$, Fan Xiang research fellow ${ }^{1}$, Anne-Louise Ponsonby professor ${ }^{2}$
}

${ }^{1}$ National Centre for Epidemiology and Population Health, Australian National University, Canberra, ACT 0200, Australia; ${ }^{2}$ Murdoch Childrens

Research Institute, Royal Children's Hospital, Melbourne, Vic, Australia

\begin{abstract}
One year ago, the chief medical officers of the United Kingdom recommended that "All pregnant and breastfeeding women should take a daily supplement containing $10 \mu \mathrm{g}$ (400 IU) of vitamin D," to counter the high prevalence of vitamin D deficiency in pregnant women. This was aimed at reducing the associated consequences of deficiency, such as rickets in children and osteomalacia in adults.

In a linked meta-analysis (doi:10.1136/bmj.f1169), Aghajafari and colleagues look beyond bone health to other adverse health outcomes for mother and baby. ${ }^{2}$ Previous systematic reviews have highlighted challenges in combining data from different studies, including diverse definitions of vitamin D deficiency, variations in vitamin $\mathrm{D}$ assays used, use of non-representative samples, and varying study designs and study quality. ${ }^{34}$
\end{abstract}

A review published in 2011 found insufficient high quality studies to conduct quantitative meta-analy $\operatorname{sis}^{3}$; in the qualitative review the evidence was inconsistent. In a subsequent review, rigorous assessment of study quality resulted in quantitative meta-analyses of only two observational studies and five randomised controlled trials, with additional studies reviewed qualitatively. ${ }^{4}$ Combined data from trials suggested that bolus high dose vitamin D supplementation (but not daily dosing) was associated with reduced risk of low birth weight (risk ratio 0.40; $95 \%$ confidence interval 0.23 to 0.71 ). Combined trial data found no significant protective effect of vitamin D supplementation on the outcome small for gestational age $(0.77$, 0.35 to 1.66 ), although observational studies supported a protective effect. Results for maternal outcomes were inconsistent. In a 2012 Cochrane systematic review, meta-analysis of three trials of daily vitamin D supplementation during pregnancy found a reduced risk of low birth weight $(0.48$, 0.23 to 1.01 ), although this was not significant. ${ }^{5}$

In a recent combined analysis of two randomised controlled trials, higher vitamin D (measured as serum concentration of 25-hydroxyvitamin D; 25-OHD) at delivery was associated with a significantly $(\mathrm{P}<0.006)$ decreased risk of "comorbidities of pregnancy." Comorbidities were gestational diabetes, hypertension, infection, bacterial vaginosis, and preterm birth without pre-eclampsia, but the study did not have enough power to analyse individual outcomes. ${ }^{6}$
Meta-analysis overcomes the problems of small sample sizes and insufficient power. But challenges arise in combining data from studies of different designs, inclusion and exclusion criteria, and definitions of exposure and outcome. Aghajafari and colleagues' review contains no primary data from vitamin D intervention studies. ${ }^{2}$ Only one trial was considered, but was excluded from analysis. The largest effect sizes derive from case-control studies, some with minimal or no adjustment for confounding; comparisons of extreme groups (such as $<50 v$ $>75 \mathrm{mmol} / \mathrm{L}$ ), so that data from most of the sample (the middle group) are omitted ${ }^{7}$; and blood sampling after "disease" onset. Serum 25-OHD concentration is labile. It depends on recent intake of vitamin D and sun exposure, both of which may change, and may even be affected by preclinical disease (disease induced vitamin $\mathrm{D}$ deficiency).

Gestational age at sampling is also relevant to causal interpretations if low vitamin D status at late sampling is linked to outcomes that are usually associated with earlier gestational onset. Aghajafari and colleagues found that "vitamin D deficiency"- -variously defined and measured at different gestational ages - is adverse for maternal and infant health. If lower vitamin $\mathrm{D}$ status causes these outcomes in a linear way, more severe deficiency $(<50 \mathrm{nmol} / \mathrm{L})$ would be expected to have a stronger effect than less severe deficiency $(<75 \mathrm{nmol} / \mathrm{L})$. The opposite effect seems to occur for pre-eclampsia. ${ }^{2}$

Despite these challenges to interpreting the evidence, these studies have clear clinical implications. In 2010 the US Institute of Medicine recommended that a serum concentration of $25-\mathrm{OHD}$ of $50 \mathrm{nmol} / \mathrm{L}$ or more should be considered sufficient for bone health. ${ }^{8}$ Although optimal maternal 25-OHD levels at different gestational times are not known, levels below 50 $\mathrm{nmol} / \mathrm{L}$ are common during pregnancy, particularly in populations at high latitudes and in specific subpopulations. Evidence of a causal association between vitamin D deficiency and some maternal and neonatal outcomes is insufficient, but the evidence for bone health is clear cut. The findings of this meta-analysis support a goal of vitamin D sufficiency for all pregnant women. ${ }^{2}$ Supplements, diet, and sunlight exposure all influence 25-OHD levels and should be used together, with care, because $\mathrm{U}$ shaped dose-response curves are reported for 
a range of health outcomes, including small for gestational age, ${ }^{9}$ with disease risk increasing at both low and high 25-OHD levels.

Most studies are undertaken in developed countries. Yet Asian and African countries have higher infant mortality and represent half of the global population. Where it has been measured, vitamin $\mathrm{D}$ deficiency is common in these countries, under the combined influences of darker skin, cultural practices that limit sun exposure, and, in some locations, urban air pollution blocking ultraviolet radiation. For example, median 25-OHD levels of pregnant women living in Beijing were only 26 $\mathrm{nmol} / \mathrm{L} .{ }^{10}$ If there is a causal association between vitamin $\mathrm{D}$ deficiency and adverse maternal and neonatal outcomes, gains from ensuring sufficiency may be great in these countries.

Current evidence on vitamin D status and neonatal and pregnancy health derives largely from observational studies, small trials, low doses of vitamin D supplementation, unclear study processes of randomisation and blinding, or low adherence. In their editorial, Harvey and Cooper called for large well designed randomised controlled trials to clarify the causal association between vitamin D supplementation and health. ${ }^{11}$ This is particularly needed to delineate the importance of vitamin $\mathrm{D}$ in pregnancy, with its potentially lifelong effects on the health of offspring. ${ }^{12}$

Competing interests: We have read and understood the BMJ Group policy on declaration of interests and declare the following interests: none.
Provenance and peer review: Commissioned; not externally peer reviewed.

1 Department of Health. Vitamin D-advice on supplements for at risk groups. 2012. www. dh.gov.uk/health/2012/02/advice-vitamin-d/.

2 Aghajafari F, Nagulesapillai T, Ronksley PE, Tough SC, O'Beirne M, Rabi DM. Association between maternal serum 25 -hydroxyvitamin $D$ level and pregnancy and neonatal outcomes: systematic review and meta-analysis of observational studies. BMJ 2013;346:f1169.

3 Nassar N, Halligan GH, Roberts CL, Morris JM, Ashton AW. Systematic review of first-trimester vitamin D normative levels and outcomes of pregnancy. Am J Obstet Gynecol 2011;205:208.e1-7.

4 Thorne-Lyman A, Fawzi WW. Vitamin D during pregnancy and maternal, neonatal and infant health outcomes: a systematic review and meta-analysis. Paediatr Perinat Epidemiol 2012;26(suppl 1):75-90.

5 De-Regil L, Palacios C, Ansary A, Kulier R, Pena-Rosas J. Vitamin D supplementation for women during pregnancy. Cochrane Database Syst Rev 2012;2:CD008873.

6 Wagner CL, McNeil RB, Johnson DD, Hulsey TC, Ebeling M, Robinson C, et al. Health characteristics and outcomes of two randomized vitamin $D$ supplementation trials during pregnancy: a combined analysis. J Steroid Biochem Mol Biol 2013; published online 10 Jan.

7 Zhang C, Qu C, Hu FB, David RM, van Dam RM, Bralley A, et al. Maternal plasma 25-hydroxyvitamin $\mathrm{D}$ concentrations and the risk for gestational diabetes mellitus. PloS One 2008;3:e3753.

8 Institute of Medicine. Dietary reference intakes for calcium and vitamin D. Ross A, Taylor CL, Yaktine AL, Del Valle HB, eds. Institute of Medicine of the National Academies, 2010. 9 Bodnar LM, Catov JM, Zmuda JM, Cooper ME, Parrott MS, Roberts JM, et al. Maternal serum 25-hydroxyvitamin $D$ concentrations are associated with small-for-gestational age births in white women. $J$ Nutr 2010;140:999-1006.

10 Wang O, Nie M, Hu YY, Zhang K, Li W, Ping F, et al. Association between vitamin D insufficiency and the risk for gestational diabetes mellitus in pregnant Chinese women. Biomed Environ Sci 2012;25:399-406.

11 Harvey NC, Cooper C. Vitamin D: some perspective please. BMJ 2012;345:e4695

12 Lucas RM, Ponsonby AL, Pasco JA, Morley R. Future health implications of prenatal and early-life vitamin D status. Nutr Rev 2008;66:710-20.

Cite this as: BMJ 2013;346:f1675

๑) BMJ Publishing Group Ltd 2013 